\title{
O PSICÓLOGO E O MORRER: COMO INTEGRAR A PSICOLOGIA NA EQUIPE DE CUIDADOS PALIATIVOS NUMA PERSPECTIVA FENOMENOLÓGICA EXISTENCIAL
}

\author{
PSYCHOLOGIST AND DYING: HOW TO INTEGRATE \\ PSYCHOLOGY IN THE PALLIATIVE CARE TEAM ON AN \\ EXISTENTIAL PHENOMENOLOGICAL PERSPECTIVE
}

\author{
José Edilmar Gonçalves ${ }^{1}$ \\ Verônica Siqueira Araújo ${ }^{2}$
}

\begin{abstract}
RESUMO: O objetivo deste estudo é fornecer elementos para o campo dos cuidados paliativos, para aprimoramento das práticas do profissional de psicologia como membro integrante da equipe que dáo suporte necessário a pacientes com diagnóstico clínico desfavorável. É um estudo que tem por base o levantamento bibliográfico. Abordou-se uma ótica multidisciplinar voltada especialmente à análise da importância do profissional psicólogo hospitalar junto aos cuidados paliativos. O material utilizado mostrou-se contundente e útil nas suas propostas e capaz de propiciar a argumentação necessária. Os Cuidados Paliativos são atualmente uma necessidade, um direito e uma responsabilidade, tanto ética quanto social, vão ao encontro às necessidades dos doentes e família, quando a terapêutica curativa já não faz sentido. Quando esta se torna fútil e desapropriada, é fundamental recorrer à terapêutica paliativa, através da qual se pretende proporcionar o maior bem-estar possivel na fase em que o doente se encontra. Este estudo reitera a importância do psicólogo dentro do contexto específico do cuidado paliativo.
\end{abstract}

Palavras-chave: Cuidados Paliativos, Psicologia, Morte, Adoecimento.

\begin{abstract}
The objective of this study is to provide the necessary elements to fill palliative care for the improvement of professional psychology practices as an integral member of the support team necessary for the treatment of patients with unfavorable clinical diagnosis. It is the study that is based on the bibliographic survey. It was approached a multidisciplinary approach aimed at the analysis of the professional hospital psychologist with palliative care. The material used was forceful and useful in its proposals and is capable of providing a necessary argument. Palliative Care is currently an obligation, a
\end{abstract}

\footnotetext{
${ }^{1}$ Graduando em Psicologia pela Faculdade de Ciências Aplicadas Dr. Leão Sampaio. Email: edmar._va@hotmail.com

${ }^{2}$ Mestre em Psicologia pela Universidade Federal do Ceará, professora do curso de Psicologia da Faculdade Leão Sampaio.
} 
right and a responsibility, just as they are social, they go to the place for the needs of the patients and the family, when it comes to healing no longer makes sense. When this becomes difficult and expropriated, it is essential to resort to palliative therapy, through the extent that one makes the greater or greater power being in the patient's stage is found. This study reiterates the importance of the psychologist within the specific context of palliative care.

Keywords: Palliative Care, Psychology, Death, Illness

\section{INTRODUÇÃO}

Com o avanço da medicina, a luta contra doenças potencialmente fatais e a própria morte tem se estendido cada vez mais, prolongando a vida, e por vezes o sofrimento, de pacientes que já não apresentam possibilidade de cura. Essa nova realidade, atrelada ao envelhecimento populacional que ocasiona o aumento das doenças crônicas, tem demandado uma busca de novas práticas pelos profissionais da área da saúde no intuito de melhorar o período final de vida do doente, sendo o psicólogo um agente ativo no que diz respeito ao alívio do sofrimento, podendo agregar qualidade à vida e ao processo de morrer. Esse modelo de atenção e cuidado com a vida e o indivíduo é conhecido como Cuidados Paliativos.

Trabalhar a questão da morte como um processo natural requer que se tenha estabelecido entre o paciente e o psicólogo um vínculo de confiança, pois as fantasias acerca deste tema e do desejo de imortalidade é o ponto primordial para a ressignificação da intensa experiência que é o processo de término da vida. Por isso o fazer psicológico na perspectiva dos cuidados paliativos solicita do profissional especial atenção à linguagem simbólica e ao não dito.

Sabe-se, desde o início da pesquisa, que não há muitas referências que especifiquem o papel do psicólogo num programa de cuidados paliativos, podendo-se ter como uma das prováveis causas o fato de que na Inglaterra, onde nasceu a filosofia dos cuidados paliativos, o psicólogo não se encontra inserido na equipe multidisciplinar (Bertan \& Castro, 2009). Dessa forma, a referida defasagem, quanto ao papel da categoria nessa questão, é um dos motivadores para a realização desta pesquisa.

Desse modo, espera-se que o estudo sobre o referido tema ofereça elementos que contribuam para o estabelecimento de diretrizes no campo psicológico dos cuidados paliativos, bem como para o aprimoramento das práticas do profissional de psicologia como membro integrante da equipa 
multiprofissional que dá o suporte necessário para se estabelecer, dentro do possível, a qualidade de vida de pacientes com prognóstico clínico desfavorável. Nesse contexto, ressalta-se a importância da manutenção do equilíbrio nas suas relações com os outros profissionais, bem como a relevância da construção de vias de comunicação que permitam a troca e a partilha do conhecimento, a partir de diferentes saberes.

Para se efetivar a prática dos cuidados paliativos é fundamental que se tenha uma abordagem multidisciplinar que produza uma assistência harmoniosa, onde o foco é amenizar e controlar os sintomas de ordem física, psicológica, social e espiritual e não de buscar a cura de determinada doença. Trata-se de oferecer ao paciente qualidade de vida, enquanto vida houver.

\section{PROCEDIMENTOS METODOLÓGICOS}

No seu significado mais simples, pesquisar corresponde a uma forma de responder indagações previamente propostas. Lakatos e Marconi (2010) apresentam a pesquisa científica como uma realização concreta de uma investigação planeada, redigida e desenvolvida por meio de normas metodológicas com vistas a alcançar os resultados.

Gil (2010) define a pesquisa sob um prisma mais pragmático, identificando-a como um processo mais formal e sistemático cujo objetivo é descobrir respostas para problemas propostos, para isso utilizando-se procedimentos científicos.

De forma geral, resume-se pesquisa como sendo um conjunto de ações com o fim único de encontrar solução para um problema, tendo como base procedimentos sistemáticos e racionais, sendo realizada quanto não se conta com informações suficientes para chegar-se a uma resposta.

Para a realização da pesquisa aqui apresentada utilizou-se como método o estudo bibliográfico, que, segundo Gil (2010), consiste numa investigação sistemática realizada com base em material publicado em livros, revistas, periódicos (físicos e/ou eletrônicos) de modo a fornecer instrumental analítico para qualquer que seja a pesquisa.

A principal vantagem deste molde de pesquisa reside no fato de permitir ao investigador a cobertura de uma gama de fenómenos muito mais ampla do que aquela que poderia pesquisar diretamente, tornando-se particularmente importante quando o problema de pesquisa requer dados muito dispersos pelo espaço (Gil, 2010). 
Tem como finalidade promover o contato direto do pesquisador com o que foi escrito sobre determinado assunto, não sendo apenas uma repetição das informações, mas galgando a possibilidade de um exame minucioso do mesmo tema sob novas perspectivas, chegando a conclusões inovadoras (Lakatos e Marconi, 2010).

$\mathrm{Na}$ recolha de materiais para esta pesquisa utilizou-se como base livros, artigos e dissertações extraídos da base de dados do Google Acadêmico e do SCIELO. Após delimitação do objeto de estudo alguns dos itens pesquisados foram descartados conforme sua relevância de conteúdo e defasagem temporal, tendo sido mantidos livros que apresentam conceitos clássicos referentes ao tema, buscando-se atualização em periódicos recentes. Abordou-se uma ótica multidisciplinar voltada especialmente para a análise da importância do profissional psicólogo hospitalar junto aos cuidados paliativos. O material utilizado mostrou-se contundente e útil em suas propostas e capaz de propiciar a argumentação necessária.

\section{CUIDADOS PALIATIVOS: CONCEITO, FILOSOFIA E PRINCÍPIOS.}

Os Cuidados Paliativos são, atualmente, uma necessidade, um direito e uma responsabilidade, tanto ética quanto social (Programa, 2010). A esperança média de vida tem aumentado e a taxa de natalidade está cada vez mais reduzida, assiste-se assim a uma inversão da pirâmide demográfica e, como consequência, tem- se uma maior necessidade de cuidados humanizados no fim de vida, respeitando os valores, princípios, padrões morais e culturais de cada ser humano.

Assiste-se a um envelhecimento da população, proporcionado pelos avanços científicos da medicina. O querer viver mais, reflete o poder de "retardar a morte" (Maciel, 2008), e como consequência verifica-se o aparecimento de doenças crônicas, evolutivas e prolongadas.

Durante o séc. XIX, grupos religiosos já se envolviam no cuidado dos moribundos (Galrica Neto, 2010), mas os cuidados paliativos nascem com Cicely

Saunders, na Inglaterra, onde fundou o primeiro "hospice", St. Christopher no ano de 1967, um serviço hospitalar cujo objetivo era cuidar e aliviar o sofrimento e a dor, onde predominava o humanismo e a espiritualidade.

Cicely Saunders cuja formação em enfermagem se deu no St. Thomas's Hospital em Londres, viu-se mais tarde obrigada a abandonar o exercício 
da profissão por problemas de saúde. Tornou-se assistente social, continuando a fazer voluntariado em enfermagem no Saint Lukes's Hospital onde mantinha o contato com doentes em fim de vida. Motivada pela sua orientação pessoal, decidida a trabalhar com doentes em fase terminal e intervir no tratamento da dor, em 1957 forma-se em Medicina (Hermes; Lamarca, 2013).

Elisabeth Kübler-Ross, Psiquiatra e Tanatologista, foi a responsável pelo movimento das unidades de cuidados paliativos nos Estados Unidos, reconhecida por seu trabalho com crianças e idosos em fase terminal e doentes com AIDS (Síndrome da Imunodeficiência Adquirida). É de sua autoria um dos mais importantes estudos psicológicos sobre a iminência da morte, a vida e a transição "On Death and Dying" (1969), com este contributo a autora descreve as reações do doente em fase terminal, assim como a compreensão global das suas necessidades e da família (KüblerRoss, 2008).

Segundo Twycross (2003), o termo paliativo deriva do latim, pallium, que significa manto, capa, expressando um propósito de proteção contra as intempéries da vida. Os cuidados paliativos pretendem "acolher" os sintomas provocados pela doença, com tratamentos que têm como finalidade promover o conforto do paciente, um cuidar mais dirigido ao doente do que à doença.

Os cuidados paliativos (C.P.) vão ao encontro às necessidades dos doentes e família, quando a terapêutica curativa já não faz sentido. Quando esta se torna fútil e desapropriada, é fundamental recorrer à terapêutica paliativa, através da qual se pretende proporcionar o maior bem-estar possível na fase em que o doente se encontra (GALRICA Galrica Neto, 2010). Para Maciel (2008) os C.P. são também uma resposta adequada, para de forma assertiva, ter o controle sobre os problemas físicos, psicológicos, sociais e espirituais, procurando melhorar a qualidade de vida do doente, tanto quanto for possível. Paliação significa alívio do sofrimento do doente. O Programa Nacional de Cuidados Paliativos (PNCP) de Portugal, plano de ação que orienta os procedimentos nessa área, e que apresenta-se como referência para vários países, define a ação paliativa como:

Qualquer medida terapêtica sem intuito curativo, que visa minorar, em internamento ou no domicílio, as repercussões negativas da doença sobre o bem-estar global, do doente. As ações paliativas são parte integrante da prática profissional, qualquer que seja a doença ou a fase da sua evolução (Programa, 2010, s.p). 
Já a Organização Mundial da Saúde (OMS) definiu Cuidados Paliativos como:

Uma abordagem que visa melhorar a qualidade de vida dos doentes que enfrentam problemas decorrentes de uma doença grave (que ameaça a vida) e a sua família, através da prevenção e alívio do sofrimento, com recurso à identificação precoce, avaliação adequada e tratamento rigoroso da dor e outros problemas físicos, psicossociais e espirituais. (2012, s.p.)

Os cuidados paliativos, ainda segundo a OMS (2012), afirmam a vida e respeitam a morte como um processo natural, não pretendem apressar nem atrasar a morte, proporcionam ao doente o alívio da dor e de outros sintomas incómodos que provocam sofrimento. Parte-se de uma perspectiva que integra os aspectos psicológicos, sociais e espirituais na assistência ao paciente, buscando-se oferecer um sistema de apoio para ajudar os pacientes a viver tão ativamente quanto possível até à morte. Utiliza uma abordagem em equipe para atender às necessidades de pacientes e das suas famílias, incluindo apoio no luto se necessário. $\mathrm{O}$ objetivo é melhorar a qualidade de vida. São cuidados aplicados no início da doença, em conjugação com outras terapias, como a quimioterapia ou radioterapia. Inclui a investigação necessária para melhor compreender e trabalhar as complicações clínicas dolorosas em fim de vida. A prestação de Cuidados Paliativos pretende sempre proporcionar a melhor qualidade de vida, segundo Maciel (2008) a qualidade de vida está relacionada com o grau de satisfação subjetiva que a pessoa sente pela vida e é influenciada por todas as dimensões da subjetividade - física, psicológica, social e espiritual.

É de grande relevância perceber o que é possível alcançar, para poder afastar as aspirações irrealistas, devendo-se sublinhar a importância da aliança terapêutica entre a equipe, o doente e a família neste processo. De acordo com Maciel (2008), existe boa qualidade de vida quando as aspirações de um indivíduo são atingidas e preenchidas pela sua situação atual. Em contrapartida, há má qualidade de vida quando há grande divergência entre as aspirações e a situação atual.

$\mathrm{O}$ conceito de qualidade de vida é subjetivo, multidimensional e transversal a todas as áreas do conhecimento (Ribeiro, 2009). Segundo o mesmo autor, uma das características fundamentais da avaliação da qualidade de vida $(\mathrm{QV})$ é o fato de ser baseada na opinião pessoal do próprio doente, o que torna evidente que as suas características psicológicas determinam a sua percepção. Considera-se, portanto, a necessidade de identificar as variáveis psicológicas que contribuem para avaliar a $\mathrm{QV}$ 
como sendo boa ou má. A subjetividade reflete o caráter pessoal do conceito de qualidade de vida, já que a avaliação pessoal é um dos seus principais critérios.

Quando se trata de cuidados paliativos, o uso da tecnologia emerge como uma questão de grande relevância. A utilização dos recursos tecnológicos deve ser avaliada com o auxílio e a prevalência da ética. Os avanços tecnológicos podem, se mal utilizados, transformarem-se em instrumentos de prolongação do sofrimento a qualquer custo, retardando o inevitável processo de morte, causando ao paciente uma agonia por métodos artificiais (Pacheco, 2004).

Os profissionais de saúde têm assim dupla responsabilidade, de defender a vida e aliviar o sofrimento. Em cuidados paliativos torna-se necessário proporcionar conforto, tornar a vida que resta ao paciente o mais suportável e significativa possível e não apressar a morte. Pacheco (2004) enuncia princípios éticos que devem servir de mediadores na prestação de cuidados paliativos, e que devem ser aplicados no âmbito do respeito pela vida e da aceitação da inevitabilidade da morte.

O cuidado da dor e do sofrimento nas suas várias dimensões, aliado ao humanismo e à competência são a base do cuidar sensível em contexto hospitalar, de forma a propiciar uma vida digna, com respeito pelos valores humanos do doente em fim de vida (Pessini, 2002).

Em cuidados paliativos é essencial uma abordagem que visa práticas de cuidados que vão ao encontro das necessidades dos doentes e familiares, uma visão colocada na melhoria da qualidade de vida, onde a qualidade no cuidar seja de excelência, para que o doente se sinta tratado como pessoa, com valor e respeito pela sua dignidade (Galrica Neto, 2010).

Segundo Pacheco (2004) a dignidade da pessoa está relacionada com a condição de liberdade do ser humano, mas também com o "[...] direito de cada homem tem de ser reconhecido como um ser que é um fim, e nunca um meio a serviço dos fins dos outros" (Pacheco, 2004, p. 23). A dignidade, enquanto liberdade, faz parte da natureza do ser humano, liberdade da pessoa consciente de si e dos seus atos, liberdade para escolher entre o bem e o mal, uma liberdade responsável, é a liberdade de caráter que leva o homem a atingir os seus propósitos. Desde o nascimento até a morte do homem a dignidade deve ser preservada (Pacheco, 2004). 


\section{PSICOLOGIA E CUIDADOS PALIATIVOS}

A promoção da saúde, do bem-estar, da qualidade de vida e da plenitude do desenvolvimento humano faz parte dos objetivos a que o exercício da psicologia se propõe. A pessoa nos seus diferentes contextos é o objeto de estudo da psicologia, tendo como principal instrumento de intervenção a relação interpessoal. O psicólogo deve perceber a pessoa, respeitar e promover a sua autonomia e sua autodeterminação, aceitando-a de forma incondicional.

Segundo Mesquita (2012), no que se refere aos seres humanos, durante o século XIX predominou o paradigma biomédico, segundo o qual o homem era visto como um ser puramente biológico. De acordo com este modelo, a doença provém do exterior, que provoca no organismo alterações biológicas que o indivíduo não controla. A saúde e a doença são vistas como extremos opostos, sendo irreconciliáveis, ou seja, para essa visão, a pessoa ou é saudável ou é doente. Ainda de acordo com este modelo, o corpo (físico) e a mente (abstrato) funcionam de forma independente.

No século XX novas abordagens compuseram o modelo Biopsicossocial. A medicina psicossomática afirmou interação entre mente e corpo, porque fatores psicológicos podem não só ser consequência de uma doença, mas também contribuir para o seu aparecimento. Também a Saúde Comportamental e a Medicina Comportamental colocaram em xeque o modelo biomédico, o papel desempenhado pelo comportamento na determinação do estado de saúde, bem como na prevenção da doença e na manutenção da saúde.

A Psicologia da Saúde surge como contributo da psicologia na compreensão da saúde. Segundo Mesquita (2012), a psicologia da saúde é o conjunto das contribuições específicas, educacionais, científicas e práticas da disciplina da psicologia para a promoção e manutenção da saúde, prevenção e tratamento da doença e disfunções relacionadas.

Segundo Mesquita, citando Watson et al. (2012), todos os profissionais devem ter uma compreensão básica de atendimento psicológico, para que a sua prática clínica seja otimizada, contudo cabe ao psicólogo, com a sua perspectiva e dotado de competências especializadas, ajudar o paciente e a sua família nas suas perdas e em seu sofrimento.

Os psicólogos têm formação no sentido de compreender o comportamento humano, têm as competências necessárias para apoiar e estimular a expressão do pensamento e emoções, no sentido de ajudar a enfrentar a doença através de suas práticas, com uma comunicação 
apropriada e desenvolvimento de estratégias próprias (Mesquita citando Watson et al., 2005).

A OMS (2012) define cuidados paliativos e menciona que estes integram o apoio psicológico no cuidado ao paciente. Os aspectos psicológicos e problemas psicossociais são elementos essenciais em cuidados paliativos, contudo o papel do psicólogo nestes serviços não é claramente definido, uma vez que todos os profissionais de saúde devem ter algum conhecimento da dinâmica psicológica na doença com prognóstico desfavorável, como habilidades de comunicação e avaliação de risco psicológico (Mesquita citando Jünger et al., 2012).

Hennezel (2001) considera que o psicólogo que trabalha numa equipe de cuidados paliativos tem como função principal dar apoio psicológico aos doentes e familiares, devendo ter a seu cargo também a formação da equipa.

O psicólogo em CP deve acompanhar o doente na sua "crise de morte". Este é um acontecimento psíquico único e singular. Assistir psicologicamente a morte como processo, exige identificar os mecanismos de defesa inconscientes, respeitando-os e ajustando-se a eles.

A manutenção ou restabelecimento da autoestima do paciente e a procura de um novo sentido para a vida é outro dos objetivos da intervenção do psicólogo, porque a doença e a iminência de morte provocam uma grande vulnerabilidade psicológica, compreendendo fases de grande angústia e desespero. Deve ser também valorizada e considerada indispensável a presença efetiva de alguém que possa controlar a angústia e aflição psíquica, identificando-as e desta forma ajudando a ultrapassá-las (Hennezel, 2001). Sobre a importância do suporte psicológico, Mesquita (2012, p. 11) afirma: “Orientado psicologicamente poderá ser possível que, apesar de dolorosa, a fase final da vida possa ter um importante e saudável balanço emocional".

O psicólogo deverá criar uma relação de confiança de forma a clarificar ou facilitar a comunicação junto daqueles com quem interage no ambiente terapêutico, no sentido de lhes diminuir a ansiedade, a depressão e o sofrimento. É o profissional privilegiado para explorar as dúvidas, as crenças, os mitos e medos do doente, assim como as suas necessidades específicas (Hennezel, 2001).

Os problemas do foro psicológico, quando não atendidos, podem resultar num maior sofrimento para o doente e familiares, assim como dificultar o controlo de outros sintomas e privar o doente de uma morte digna. O psicólogo é também responsável por detectar sinais de alarme e prevenir possíveis doenças da equipa, que, por lidar com situações de 
grande stress está mais vulnerável a transtornos tais como o burnout. Este último é caracterizado por ser o ponto máximo do stress profissional, podendo ser encontrada em variadas profissões, mas em especial nos trabalhos em que há impacto direto na vida de outras pessoas (Lacasta, 2008).

O acompanhamento, o diálogo e a atenção desempenham uma importância particular no doente em fim de vida. Assim, o psicólogo deve estabelecer a conveniente relação de ajuda. Para Rogers (2001) a relação de ajuda é uma relação na qual pelo menos uma das partes procura promover na outra o crescimento, assim como o desenvolvimento, a maturidade, um melhor funcionamento e uma maior capacidade de enfrentar a vida.

No entendimento de Mesquita (2012), a relação de ajuda na fase final de vida deve ser proporcionada por um profissional, alguém que conheça o conteúdo da ajuda a outro ser humano que necessita desse suporte. $\mathrm{O}$ importante nessa relação é o saber ser e o saber estar com o doente e não temer a morte, devendo assim evitar os próprios mecanismos de defesa e cultivar os três fundamentos da relação de ajuda: o respeito, a verdade e o amor.

Neste sentido, a comunicação deve ser apoiada em três componentes: 1) A escuta ativa que consiste em mostrar interesse e atenção no que o paciente diz, nesse sentido, fomentar a sua expressão ajuda na verbalização de um problema ou conflito e em seu esclarecimento, sendo fundamental nos cuidados paliativos; 2) A empatia, entendida como a capacidade de se colocar no lugar da outra pessoa; 3) A aceitação do paciente e daquilo que ele diz. Tal aceitação confere ao indivíduo confiança e abertura para expressar os seus sentimentos. A sensibilidade, a preocupação genuína e a comunicação não verbal são capacidades basilares em cuidados paliativos na atenção ao doente e à sua família.

Mesquita (2012) sustenta ainda que a comunicação é um pilar fundamental em CP. Ela deve basear-se na verdade, na confiança mútua e na compreensão. Os objetivos desta são: informar, orientar e apoiar.

O psicólogo deve estar atento em detectar os conteúdos envolvidos na queixa, no sintoma e na patologia, permitindo assim uma atenção integral e a identificação de desordens psíquicas que geram sofrimento e estresse. Deve estar atento ainda aos mecanismos de defesa negativos que costumam surgir; isso favorece a reorganização da vivência da doença e o uso de recursos adaptativos no sentido de manter o paciente participativo no tratamento (Ferreira \& Lopes \& Melo, 2011). 
Conforme Ferreira \& Lopes \& Melo, (2011) citando Sampaio e Löhr:

(...) A psicologia é uma das profissões da saúde cuja inclusão em equipes de acompanhamento de pacientes com câncer é regulamentada por lei. A Portaria $\mathrm{n}^{\circ} 3.535$ do Ministério da Saúde, publicada no Diário Oficial da União em 14 de outubro de 1998, determina que toda equipe responsável pelo tratamento de pessoas com câncer tenha, entre seus profissionais, um psicólogo (SAMPAIO \& LÖHR, 2008, p.56).

Dar assistência aos pacientes envolve, sobretudo, integrar as várias dimensões do ser, isto inclui também o aspecto espiritual. Estudos mostram que as questões referentes à espiritualidade representam para o paciente em cuidados paliativos, na sua maioria, uma fonte de conforto, fé e suporte para enfrentamento da doença, apresentando-se como fator de contribuição na adesão ao tratamento (Fornazari \& Ferreira, 2010). Portanto, para o psicólogo é fundamental perceber o fenômeno espiritual como um recurso que possibilite buscar alternativas para reforçar o suporte emocional do paciente, proporcionando entre outras coisas, sentido à vida e ao sofrimento humano presente no processo de adoecimento (Silva, 2010).

Trabalhar a questão da morte, como um processo natural, requer que se tenha estabelecido entre o paciente e o psicólogo um vínculo de confiança, pois as fantasias acerca deste tema e do desejo de imortalidade é o ponto primordial para a ressignificação da intensa experiência que é o processo de término da vida. Dessa forma, o fazer psicológico na perspectiva dos cuidados paliativos solicita do profissional especial atenção à linguagem simbólica e ao não dito (Kovács, 2006).

Para além da intervenção técnica, também devem estar presentes no trabalho do psicólogo a empatia e a escuta acolhedora verbal e não-verbal, permitindo que o paciente possa confrontar-se com seus conteúdos internos, suas angústias e sentimentos em geral, para que a partir daí inicie o processo de aceitação, elaboração e superação no que diz respeito ao adoecimento. A escuta permite ao psicólogo identificar as reais demandas do paciente (Othero \& Costa, 2007).

\section{CONSIDERAÇÕES FINAIS}

Por meio da discussão estabelecida relacionada aos Cuidados Paliativos (CP), pode inferir-se a importância da inserção da Psicologia na equipa multidisciplinar que oferece tais cuidados. O trabalho do psicólogo é imprescindível, uma vez que, ao mesmo tempo que busca aliviar o 
sofrimento emocional de todos os envolvidos no processo, trabalha com o paciente em prol da qualidade de vida e melhor aceitação de sua condição.

Um dos objetivos primordiais do atendimento psicológico é mostrar ao paciente que o momento vivido pode ser compartilhado. Assim, estimulase a busca de seus recursos internos, para assim atenuar sentimentos como solidão e derrota. Trabalha-se com o paciente o seu sofrimento psíquico, que pode incluir ansiedade, depressão, perda da dignidade e medos, em um compartilhar de cumplicidade capaz de favorecer a ressignificação desta experiência que é o adoecer e o morrer.

Tendo cuidado para não ocupar o lugar de mais um elemento invasivo no tratamento, mas de facilitador no processo de integração do paciente, da família e da equipe multidisciplinar, o profissional da psicologia deve manter como foco o doente e não a doença, e a melhora na qualidade de vida do paciente e não o prolongamento infrutífero do seu sofrimento.

O presente estudo procorou destacar, assim, a importância do psicólogo integrado na equipe multidisciplinar em Cuidados Paliativos, enfatizando a sua atuação como promotor e facilitador na melhoria da qualidade de vida de pacientes com prognóstico clínico desfavorável. Nesse sentido, a psicologia mostra-se essencial na redução de agentes estressores que geram sofrimento e angústia aos pacientes e familiares. Esta pesquisa não pretendeu esgotar toda a discussão sobre o assunto, nem mesmo dar respostas únicas e fechadas para a temática, mas sim proporcionar subsídios para novas pesquisas e discussões sobre o tema.

\section{REFERÊNCIAS BIBLIOGRÁFICAS}

ANCP. Academia Nacional de Cuidados Paliativos (2009). Organização de serviços de cuidados paliativos: recomendações da ANCP. Rio de Janeiro: Diagraphic.

Bertan, F. C. \& Castro, E. K (2009). Qualidade de vida e câncer: revisão sistemática da literatura. Revista Psico, 40, 366-372.

Ferreira, A.P., Lopes, L. Q., Melo, M, C (2011). O papel do psicólogo na equipe de cuidados paliativos junto ao paciente com câncer. Rev. SBPH vol.14 no.2, Rio de Janeiro - Jul/Dez.

Fornazari, S. A.; Ferreira, R. E. R. Religiosidade/Espiritualidade em pacientes oncológicos: qualidade de vida e saúde. Psicologia: Teoria e papel da psicologia e construindo novos caminhos. RUBS, 1 (3), 5260, Curitiba, Set./Dez, 2008. 
Galrica Neto, I. (2010). Pequeno manual básico de cuidados paliativos Região de Saúde de Lisboa. Lisboa: 2010.

Gil, A. C. (2010). Como Elaborar Projetos de Pesquisa. 5. ed. São Paulo: Atlas, 2010.

Hennezel, M. (2001) "O Papel do Psicólogo" in Abiven, Maurice (ed.) Para Uma Morte Mais Humana - Experiência de uma Unidade Hospitalar de Cuidados Paliativos (2 edição). Loures: Lusociência, 133-154, 2001.

Hermes, H. R; Lamarca, I. C. A. L. (2013). Cuidados paliativos: uma abordagem a partir das categorias profissionais de saúde. Ciênc. saúde coletiva vol.18 no.9 Rio de Janeiro Sept. 2013.

Kovács, M. J. (2006). Comunicação em cuidados paliativos. In: C. A. M. Pimenta; D. D. C. F.

Kübler-Ross, E. (2002). Sobre a morte e o morrer: o que os doentes terminais têm para ensinar a médicos, enfermeiras, religiosos e aos seus próprios parentes. São Paulo: Martins Fontes, 2002.

Lacasta, M. (2008). "Los Psicólogos en los Equipos de Cuidados Paliativos en el Plan Integral de la Comunidad de Madrid”. Psicooncología. 5(1), 171- 177.

Lakatos, E. M.; Marconi, M. A. (2010). Fundamentos de metodologia científica. 5. ed. - São Paulo: Atlas.

Maciel, M. G. S. (2008). Definições e princípios. In R. A. Oliveira (Org.), Cuidado paliativo (pp. 15-32). São Paulo: Cremesp.

Mesquita, A.S.L. (2012). O psicólogo em cuidados paliativos: intervenção em fim de vida. Dissertação (Mestrado) - Curso de Cuidados Paliativos, Faculdade de Medicina da Universidade do Porto, Porto.

Organização Mundial de Saúde [OMS] (2012). Definições e princípios. In R. A. Oliveira (Org.).

Othero, M.B.; Costa, D.G. (2007). Propostas desenvolvidas em cuidados paliativos em um hospital amparador - Terapia Ocupacional e Psicologia. Prat. Hosp., v.9, n.52, p.157-60. Disponível em: $<$ http://www.praticahospitalar.com.br/>.

Pacheco, S. (2004). Cuidar da pessoa em fase terminal: perspectiva ética. $2^{a}$ ed. Loures: Lusociência.

Pessini, L. (2006). Bioética e cuidados paliativos: alguns desafios do cotidiano aos grandes dilemas. In: C. A. M. Pimenta; D. D. C. F. Mota; D. A. L. M. Cruz (Org.), Dor e cuidados paliativos: enfermagem, medicina e psicologia (p. 4566). Barueri: Manole. 
Programa Nacional de Cuidados Paliativos (2010). Disponível em:

$<$ http://cuidandojuntos.org.pt/wpcontent/uploads/2015/09/programanacionalcuidadospaliativos.pdf>

Rezende, L. Gomes, C. Machado, M. (2014). A finitude da vida e o papel do psicólogo: perspectivas em cuidados paliativos. Revista Psicologia e Saúde, v. 6, n. 1, jan. /jun.

Ribeiro, J. (2009). A importância da qualidade de vida para a psicologia da saúde. In: J.P.Cruz, S.N. de Jesus, \& C Nunes (Coords.). Bem-Estar e Qualidade de Vida (pp.31-49). Alcochete: Textiverso.

Rogers, Carl R. (2001). Tornar-se pessoa. 5. ed São Paulo: Martins Fontes.

Sampaio, A. S.; Löhr, S. S. (2008). Atuação em casas de apoio: pensando o papel da psicologia e construindo novos caminhos. RUBS, 1 (3), 5260, Curitiba, set./Dez.

Silva, K. S. (2010). Em defesa da sociedade: a invenção dos cuidados paliativos. Dissertação de Mestrado, Universidade Federal do Rio Grande do Sul, Porto Alegre,.

Twycross, R. (2003). Cuidados paliativos. Lisboa: Climepsi. 